\title{
The Inspection on Obstructive Sleep Apnea Severity Detection using a Deep Learning Access
}

\section{N. .Iuber Rahman . P. Nithva}

\begin{abstract}
As of late, crucial endeavors are created to research thoroughgoing sleep observant to anticipate sleeprelated clutters. variable sleep organize grouping has attained unbelievable enthusiasm among specialists in well-being information science. A soft induction framework is received to assess the division of sleep organize. At that time, a starter sleep profundity is decided. Besides, a restricted state machine is made to tell apart the sleep stage changes. the excellence between our examination and alternative existing investigations is that, first, each the load sensors and also the pulse device area unit utilized; at that time, the soft induction and a restricted state machine area unit bestowed, that provide United States of America the next truth than the standard techniques to assess the sleep prepare. preventive sleep disorder (OSA) may be a typical sleep issue caused by abnormal reposeful. The seriousness of OSA will prompt various aspect effects, as an example, fulminant viscus death (SCD). Polysomnography (PSG) may be a very best quality level for OSA analysis. It records various sign from the patient's body for in any event one entire night and figures the ApneaHypopnea Index (AHI) that is that the amount of symptom or respiration occurrences each hour. This value is then accustomed prepare patients into OSA seriousness levels. The principle focal points of our projected technique incorporate easier data acquisition, prompt OSA seriousness recognition, and undefeated part extraction while not space learning from ability. Programmed sleep-organize arrangement models were worked with sturdy and explainable AI techniques (support vector machine and call tree).
\end{abstract}

Keywords: Deep Brain Stimulation (DBS), Obstructive sleep apnea (OSA), Apnea-Hypopnea Index (AHI)

\section{I.INTRODUCTION}

Sleep is a characteristic resting condition of body and brain, which covers around 33\% of human life expectancy. Because of the expanding weights of work and undesirable way of life, sleep unsettling influences become one of the genuine medical issues in current social orders. So as to lead far reaching physiological checking, medium-term polysomnography (PSG) chronicles are frequently used to break down complex physiologic occasions during sleep [1].

Deep brain stimulation (DBS) has turned into a settled careful treatment for Parkinson's sickness (PD) [6]. Nonetheless, the current generally utilized consistent high recurrence stimulation (HF-DBS) isn't adequate to satisfy developing clinical needs of increasingly exact neuro modulation to improve clinical results. Sleep issue are usually seen in phase descriptors PD patients.

Revised Manuscript Received on July 18, 2019.

N. Juber Rahman, Research Scholar, Department of Computer Science, PSG College of Arts \& Science, Coimbatore, Tamil Nadu, India.

Dr. P. Nithya, Associate Professor, Department of Computer Technology, PSG College of Arts \& Science, Coimbatore, Tamil Nadu, India.
In clinical practice, the gathered PSG records are divided into 30-second openings and outwardly examined by wellprepared specialists. In any case, long haul PSG visual assessment is incredibly tedious and relentless for doctors, and requires exceedingly prepared experts to analyze sleep issues. Consequently, it has inspired scientists to create programmed sleep organize arrangement frameworks that can effectively perform PSG sleep investigation. As of late, an assortment of strategies has been explored for the grouping of sleep stages utilizing the PSG information [2], [3]. To total and

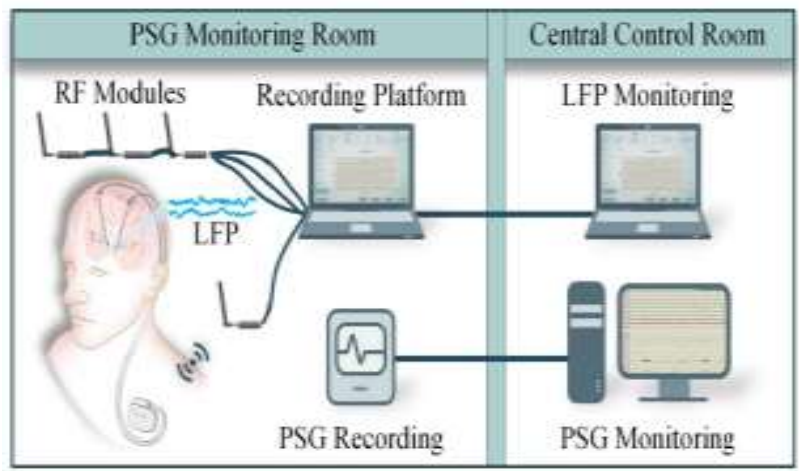

Fig 1: Sleep Monitoring System

All the more as of late, so as to consequently learn important portrayals for such multivariate biosignals, huge endeavors have been made to investigate highlight extraction procedures utilizing deep learning techniques.

In the errand of sleep arrange order, the highlights separated by deep learning models, for example, deep conviction systems, convolutional neural systems and intermittent neural systems, have demonstrated to be more powerful than the handmade highlights because of better grouping execution.

Regardless of numerous deep learning examinations revealing promising outcomes in sleep arrange characterization, a few provokes still should be tended to [1]. One of the significant difficulties is that most deep learning models neglect to unequivocally join the inalienable relationships of multivariate biosignals. On one hand, there exist complex connections among PSG channels which ought to be caught to distinguish sleep stages. Then again, the dynamic connections among the information crosswise over various timestamps (i.e., spaces) are likewise vital to catching sleeprelated occasions. Also, the shrouded

Published By: 


\section{THE INSPECTION ON OBSTRUCTIVE SLEEP APNEA SEVERITY DETECTION USING A DEEP LEARNING ACCESS}

examples during sleep change essentially crosswise over people, rendering it a moving undertaking to build up a cross-subject sleep organize classifier.

The system of HybridAtt is introduced in Fig. 1. In particular, to take in enlightening highlights from the heterogeneous PSG inputs, we first build a multi-see convolutional encoder to concentrate highlights from both channel-explicit and worldwide points of view, alluded to as channel-see and worldwide view highlights, separately. In view of the educated multi-see highlights, we at that point build up another combination based half and half consideration instrument, which comprises of a channelwise consideration layer and a timewise consideration layer, to display the double connections of PSG channels and timestamps. At long last, we receive a softmax layer utilizing the acquired attentional concealed portrayal to prepare our proposed start to finish deep learning model as a crosssubject classifier.

\section{SURVEY WORK}

Yuan, Y., Jia, K., Ma, F., Xun, G., Wang, Y., Su, L., \& Zhang, A. [1] aforesaid variable sleep organize arrangement has changed into a hot analysis theme in associate assortment of restorative applications in welfare. during this paper, we have a tendency to propose a combination selfmindful deep learning system, named HybridAtt, to order sleep phases of heterogeneous PSG records. The projected HybridAtt could be a begin to end model that consolidates multi-see convolutional portrayal with 0.5 and 0.5 self thought instrument to get rid of delegate highlights from variable biosignals. thus on unharness the intensity of multisee highlight learning, we develop another CNN structure to take in inert portrayals from channel-explicit and worldwide perspectives.

Li, X., Cui, L., Tao, S., Chen, J., Zhang, X., \& Zhang, G.Q. [2] projected a hybrid classifier (HyCLASSS), change of integrity the benefits of capricious timberland classifier and rectification tips has been projected for programmed sleep stage rating with single channel graphical record chronicles. a couple of highlights from transient, repeat and nonlinear examinations gave profitable information to portray sleep stages. Irregular backwoods classifiers were incontestable to be a valuable equipment to accumulate high exactitude sleep organize order from graphical record recordings.

Li, Y., Pan, W., Li, K., Jiang, Q., \& Liu, G.-Z. [3] projected strategy, a full night of cardiogram chronicles of seventy OSA and normal subjects were downloaded from the PhysioNet information of the Computers in medical specialty 2000 Challenge. during this projected, every chronicle incorporated plenty of reference comments for of symptom throughout that minute. Phan, H., Andreotti, F., Cooray, N., Chen, O. Y., \& De Vos, M. [4] projected a CNN structure to manage the joint issue. In spite of the actual fact that the projected system is standard as in any CNN will slot in, we have a tendency to utilize a simple CNN style with time-recurrence image input. The effectiveness of this style for programmed sleep composition was exhibited in our past work. To suit the trip of joint grouping and forecast, we have a tendency to follow the CNN's accepted softmax layer with a perform varied tasks softmax layer and gift the systematically to demonstrate the distance or nonattendance

perform varied tasks misfortune work for system making ready.Ye, J. H., Lin, Y., Li, Z. W., Lee, J., Al-Ahmari, A., \& Jin, M. [5] present a non-intrusive sleep investigation approach, which may assist U.S. with evaluating the final nature of sleep. Through the flossy derivation, we will get a primer sleep profundity by upgrading the division of sleep organize. At that time, a restricted state machine is planned to assess the sleep organize changes. Trial results demonstrate that the sleep investigation ends gotten by the planned methodology square measure steady with the PSG take a look at. In future, a lot of principles are ventured into the flossy guideline base and a few distinctive rectification parameters are conferred in Section III-H once a natural action is denied by the restricted state machine. Dynamic parameters will modify U.S. to seek out the subsequent wise state modification but very much like may fairly be expected, instead of basically getting rid of all the gathered qualities.

Chen, Y., Gong, C., Hao, H., Guo, Y., Xu, S., Zhang, Y., Li, L.[6] planned associate degree economical strategy for the grouping of sleep stages captivated with STN-LFP in metal patients. The order models will accomplish high cross-approved execution at each gathering level and individual level, while not losing interpretability and generalizability. The explicable model structure options that beta, alpha, and gamma teams contribute the foremost to arrangement, creating a stride towards revealing the neuromodulation elements of DBS and sleep issue.

\section{OBSTRUCTIVE SLEEP APNEA SEVERITY DETECTION}

In this area, we initially present our information preprocessing strategies. At that point, k-crease crossapproval has been connected to test our primary classifier. The structure of our classifier will be depicted toward the finish of this segment.

\section{1) K-fold cross-validation}

We utilized 10-overlap cross-approval by isolating examples into 3 sets including $80 \%, 10 \%$ and $10 \%$ for preparing, approval, and testing, separately. Initially, 1000 examples were chosen from each gathering of patients, and we haphazardly

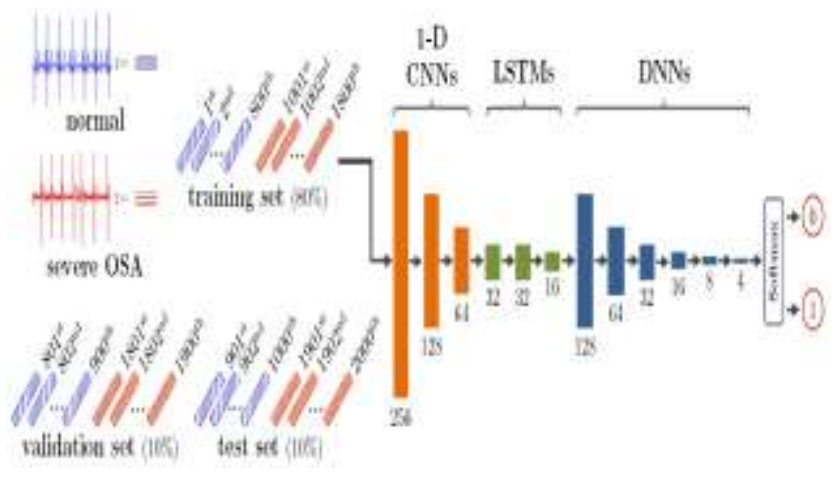

Fig 2: The structure of proposed OSA severity classifier using a Deep Learning approach 
2) OSA severity classification using Support Vector Machine

To contrast and our principle classifier, we recognized RR Intervals and ECG determined breath (EDR) signals from 15 seconds of ECG tests and removed highlights from them which are generally utilized in a few works

3) OSA severity classification using a Deep Learning (DL) approach

A heap of one-dimensional Convolutional Neural Networks (1-D CNNs) with 256, 128 and 64 units, separately, for programmed highlight parceled them into 10 equivalent measured subsamples to such an extent that there were 10 subsamples with 100 examples in each. For each subsample, it at that point stays 900 examples from the aggregate.

extraction [20]. Each CNN layer is trailed by group standardization; the amended straight unit initiation work just as the maximum pooling process with pool size equivalent to 2 so as to separate just significant highlights from the yield of its past layer. After model improvement, we assessed our primary classifier utilizing exactness, particularity, affectability and F-score. We additionally contrasted those measurements and the SVM classifier and performed a matched example t-test between two classifiers.

\section{COMPARATIVE ANALYSIS OF SURVEY}

Regularly the proposed sort is made by essentially considering the numerous downsides of the current systems administration correspondence. A large number of the prominent dynamic works all through cloud PC alongside their Limits are further down.

\begin{tabular}{|c|c|c|c|}
\hline Fupe Xam & Metudelog! & Liniatiens & $\begin{array}{l}\text { Paper } \\
\text { Sember. }\end{array}$ \\
\hline 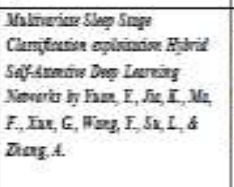 & 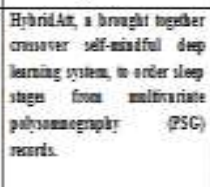 & 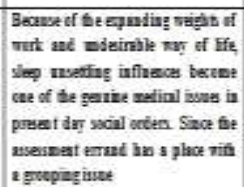 & 明 \\
\hline 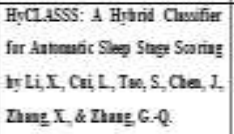 & 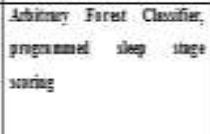 & 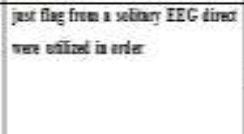 & {$[\mathrm{R}]$} \\
\hline 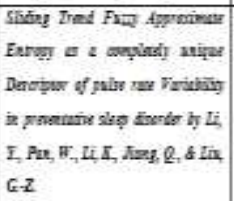 & 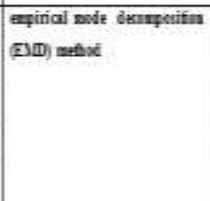 & 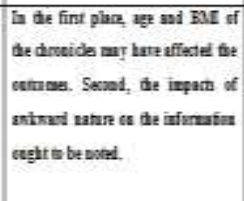 & [अ] \\
\hline 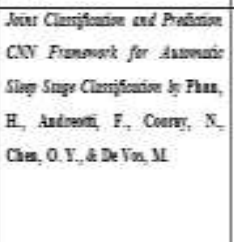 & 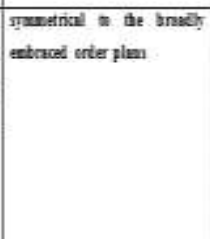 & 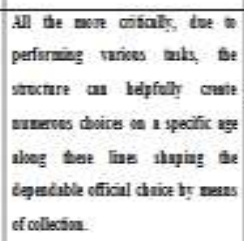 & [H] \\
\hline 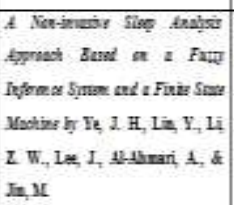 & 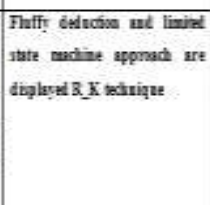 & 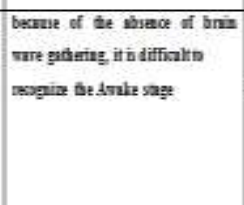 & [5] \\
\hline
\end{tabular}

Table 1: Evaluation on various authors views

\section{DISCUSSION \& RESULTS}

The objective of this examination is to research the most reasonable lead framework for OSA identification utilizing just ECG. The proposed cathode situations are assessed in the accompanying two different ways. In the first place, to survey the estimation exactness for the espiration, we figure the relationship coefficient between the real respiratory rates and those acquired by the PCA. In this paper, the relationship of the highorder measurements based respiratory rate isn't indicated on the grounds that strategy did not choose the respiratory rate of the high insights based EDR.

Next, the gatherings of eupneas and pseudo-apneas are ordered utilizing the LDA (linear discriminant analysis) in each channel. The assessment lists are the precision (TP + $\mathrm{TN} / \mathrm{TP}+\mathrm{TN}+\mathrm{FP}+\mathrm{FN})$, affectability $(\mathrm{TP} / \mathrm{TP}+\mathrm{FN})$, and particularity (TP/TP + FP). TP, TN, FP, and $\mathrm{FN}$ the quantities of true positives, true negatives, false positives, and false negatives, respectively. For the assessment, half of the 450 datasets are haphazardly chosen as learning information for LDA, and the rest are utilized as test sets.

\section{CONCLUSION}

The objective of this investigation was to come to a decision the foremost affordable EKG terminal arrangements for utilizing EDR to acknowledge OSA; 9 anode things from the customary lead frameworks were displayed. To assess the terminal positions, puffy and pseudo-apneic assignments were directed utilizing 9 anodes, and EDRs were evaluated utilizing the PCA and highrequest statistics based mostly ways. To cluster the puffy and pseudoapneic EDR waveforms, the EDR's region, most extreme kurtosis, and breath rate chosen by the technique were used as highlights. Thus, 3 cathodes settled at the lower pectoral scene were found to yield more and more precise estimations of metabolism rates and grouping exactnesses than completely different anodes. during this paper, the OSA seriousness classifier utilizing a Deep Learning methodology is projected. we have a tendency to used simply fifteen seconds of EKG with symptom or breathing occasions from 545 subjects and sustained them into a pile of CNNs, LSTMs (Long short Memory), and DNNs to cluster into two categories as well as normal and extreme OSA subjects. we have a tendency to assess our projected classifier by a great deal of measurements even as correlation with the SVM classifier with a great deal of highlights got from RR Intervals and EKG inferred breath (EDR) signal. The projected classifier is supplied for recognizing astonishingly serious OSA subjects from typical subjects with a exactness level of seventy nine. $45 \%$ that is altogether superior to the end result from the SVM classifier that has associate degree truth of fifty five.94\%. It on these lines offers associate degree beginning to future advancement of OSA seriousness discovery frameworks thus on inform clinicians quickly once extreme OSA is found. 


\section{REFERENCES}

1. Yuan, Y., Jia, K., Ma, F., Xun, G., Wang, Y., Su, L., \& Zhang, A. (2018). variable Sleep Stage Classification Mistreatment Hybrid Self-Attentive Deep Learning Networks. 2018 IEEE International Conference on Bioinformatics and Biomedicine (BIBM).

2. Li, X., Cui, L., Tao, S., Chen, J., Zhang, X., \& Zhang, G.Q. (2018). HyCLASSS: A Hybrid Classifier for Automatic Sleep Stage grading. IEEE Journal of medical specialty and Health science, 22(2), 375-385.

3. Li, Y., Pan, W., Li, K., Jiang, Q., \& Liu, G.-Z. (2018). slippy Trend Fuzzy Approximate Entropy as a completely unique Descriptor of vital sign Variability in impeding apnea. IEEE Journal of medical specialty and Health science, $1-1$.

4. Phan, H., Andreotti, F., Cooray, N., Chen, O. Y., \& De Vos, M. (2018). Joint Classification and Prediction CNN Framework for Automatic Sleep Stage Classification. IEEE Transactions on medical specialty Engineering, 11.

5. Ye, J. H., Lin, Y., Li, Z. W., Lee, J., Al-Ahmari, A., \& Jin, M. (2018). A Non-invasive Sleep Analysis Approach supported a Fuzzy logical thinking System and a Finite State Machine. IEEE Access, 1-1.

6. Chen, Y., Gong, C., Hao, H., Guo, Y., Xu, S., Zhang, Y., ... Li, L. (2019). Automatic Sleep Stage Classification supported Subthalamic native Field Potentials. IEEE Transactions on Neural Systems and Rehabilitation Engineering, 1-1.

7. Banluesombatkul, N., Rakthanmanon, T., \& Wilaiprasitporn, T. (2018). Single Channel ECG for impeding apnea Severity Detection employing a Deep Learning Approach. TENCON 2018 - 2018 IEEE Region ten Conference. 